\title{
Quasi-invariant measures on the path space of a diffusion
}

\author{
Denis Bell ${ }^{1}$ \\ Department of Mathematics, University of North Florida, \\ 4567 St. Johns Bluff Road South, Jacksonville, FL 32224, U. S. A. \\ email: dbell@unf.edu, phone: 904-620-2653, fax: 904-620-2818.
}

\begin{abstract}
The author has previously constructed a class of admissible vector fields on the path space of an elliptic diffusion process $x$ taking values in a closed compact manifold. In this Note the existence of flows for this class of vector fields is established and it is shown that the law of $x$ is quasi-invariant under these flows.
\end{abstract}

\section{Résumé}

L'auteur a précédemment construit une classe de champs de vecteurs admissibles sur l'espace des chemins d'une diffusion elliptique $x$ prenant valeurs dans une variété compacte fermée. Dans cette Note l'existence des flots pour cette classe de champs de vecteurs est établie et on montre que la loi de $x$ est quasi-invariante sous ces flots.

\footnotetext{
${ }^{1}$ Research partially supported by NSF grant DMS-0451194.
} 


\section{Introduction}

Let $N$ denote a smooth manifold equipped with a finite Borel measure $\nu$. A vector field $Z$ on $N$ is said to be admissible (with respect to $\nu$ ) if there exists an $L^{1}(\nu)$ random variable $\operatorname{Div}(Z)$ such that the equality

$$
E[Z(\Phi)(x)]=E[\Phi(x) \operatorname{Div}(Z)]
$$

holds for a dense class of real-valued functions $\Phi$ on $N$. Suppose $\left\{x \mapsto x^{s}, s \in \mathbf{R}\right\}$ is the flow on $N$ generated by $Z$. Assume $x^{0}$ is a random variable with law $\nu$ and let $\nu^{s}$ denote the law of $x^{s}$. Then $\nu$ is said to be quasi-invariant under the flow of $Z$ if $\nu_{s}$ and $\nu$ are equivalent measures, for all $s$.

These two properties are closely related. For example, suppose $\nu$ is quasi-invariant under the flow of a vector field $Z$ and write $\rho_{s}$ for the family of Radon-Nikodym derivatives $\rho_{s}=\frac{d \nu_{s}}{d \nu}$. Then an obvious calculation suggests that $Z$ will generally be admissible, with

$$
\operatorname{Div}(Z)=\frac{d \rho_{s}}{d s} / s=0
$$

The converse is not necessarily true, as is easily seen by considering the case where $\nu$ is a measure on a Euclidean space with a smooth compactly supported density and $Z$ is a constant vector field. However, admissibility of $Z$ with respect to $\nu$, together with the existence of a suitably regular version of the process $\operatorname{Div}(Z)\left(x^{s}\right)$, can be shown to imply quasi-invariance of $\nu$ under the flow $x^{s}$ of $Z$ (cf. Theorem 3 below).

In this Note we study these properties in the following setting. Let $X_{1}, \ldots, X_{n}$ and $Y$ denote smooth vector fields defined on a closed compact $d$-dimensional manifold $M$. Consider the Stratonovich stochastic differential equation (SDE)

$$
\begin{aligned}
d x_{t} & =\sum_{i=1}^{n} X_{i}\left(x_{t}\right) \circ d w_{i}+Y\left(x_{t}\right) d t, \quad t \in[0, T] \\
x_{0} & =o
\end{aligned}
$$

where $\left(w_{1}, \ldots, w_{n}\right)$ is a standard Euclidean Wiener process and $o$ is a point in $M$. Assume the diffusion (1) is elliptic, i.e. the vector fields $X_{1}, \ldots, X_{n}$ span $T M$ at each point of $M$. Define $\nu$ to be the law of $x$, considered as a measure on $C_{o}(M)$, the space of continuous paths $\{\sigma:[0, T] \mapsto M / \sigma(0)=o\}$.

The author ([1] and [2]) has recently constructed a new class of admissible vector fields on the path space $\left(C_{o}(M), \nu\right)$. These vector fields, and their divergences, are described in Theorem 1. Theorem 2 asserts that the vector fields in Theorem 1 generate flows on $C_{o}(M)$. Theorem 3 gives a criterion under which admissibility of a vector field implies quasi-invariance of the corresponding flow of measures. The main result in the paper is Theorem 4, which asserts that the measure $\nu$ is quasi-invariant under the flows generated by the vector fields in Theorem 1. 
The issue of quasi-invariance of measures under flows of vector fields has been well-studied in the the past two decades. Significant results in this area have been obtained by Driver [5] and Hsu [6] in the classical path space framework studied here and by Cruzeiro [4] for vector fields on abstract Wiener space. The results presented here are a continuation of this tradition.

\section{Additional notations}

Denote by $\left[g_{j k}\right]_{j, k=1}^{d}$ the Riemannian metric on $M$ defined by $g^{j k}=a_{i j} a_{i k}$ where $X_{i}=a_{i r} \partial / \partial x_{r}$ is the local representation of $X_{i}, 1 \leq i \leq n$ (here and henceforth, we adopt the usual summation convention: whenever an index in a product is repeated, that index is assumed to be summed on). Let $\nabla$ denote the Levi-Civita covariant derivative associated with this metric.

Define a set of 1 -forms $\omega^{j k}, 1 \leq j, k \leq n$ on $M$ by

$$
\omega^{j k}(.)=<\nabla_{X_{j}} X_{k}, .>-<\nabla . X_{j}, X_{k}>
$$

and functions $B^{j k}, 1 \leq j, k \leq n$ on $M$ by

$$
\begin{array}{r}
B^{j k}(x)=\frac{1}{2}\left(<L_{j i} X_{i}, X_{k}>-<L_{i j} X_{k}, X_{i}>-<\nabla_{X_{j}} X_{k}, \nabla_{X_{i}} X_{i}>\right. \\
\left.+<\nabla_{X_{p}} X_{i}, X_{k}><\nabla_{X_{j}} X_{p}, X_{i}>\right)(x)
\end{array}
$$

where $L_{i j}$ is the differential operator $\nabla_{X_{i}} \nabla_{X_{j}}-\nabla_{\nabla_{X_{i}} X_{j}}$.

\section{Statement of results}

The methods of [1] and [2] yield the following

Theorem 1 Let $r=\left(r^{1}, \ldots, r^{n}\right)$ be any path in the Cameron-Martin space of $\mathbf{R}^{n}$ and define $h^{i}, 1 \leq i \leq n$ by the following system of SDE's

$$
\begin{aligned}
d h_{t}^{i} & =\omega^{j i}\left(\circ d x_{t}\right) h_{t}^{j}+\left[B^{j i}+<\nabla_{X_{j}} Y, X_{i}>\right]\left(x_{t}\right) h_{t}^{j} d t+\dot{r}_{t}^{i} d t \\
h_{0}^{i} & =0 .
\end{aligned}
$$

Then the vector field $Z(x)_{t} \equiv X_{i}\left(x_{t}\right) h_{t}^{i}, t \in[0, T]$ on $C_{o}(M)$ is admissible and

$$
\operatorname{Div}(Z)=\int_{0}^{T}\left(\dot{r}_{t}^{i}+\frac{1}{2}<\operatorname{Ric}\left(Z_{t}\right), X_{i}\left(x_{t}\right)>\right) d w_{i}
$$

where Ric denotes Ricci curvature.

Although the equations defining $B^{j k}$ are lengthy, the form of the vector field $Z$ is relatively simple. The next result establishes the existence of flows on $C_{o}(M)$ for vector fields of this form. 
Theorem 2 Let $T^{i j}$ and $f^{i j}, 1 \leq i, j \leq n$ be, respectively, smooth 1-forms ond real-valued functions on $M$ and suppose $g^{i} \in L^{2}[0, T], 1 \leq i \leq n$ are deterministic real-valued functions. Define a vector field $V$ on $C_{0}(M)$ by $V_{t}=X_{i}\left(x_{t}\right) \eta_{t}^{i}$, where $\eta_{1}, \ldots, \eta_{n}$ solve the system of $S D E$ 's

$$
\begin{aligned}
d \eta_{t}^{i} & =T^{i j}\left(\circ d x_{t}\right) \eta_{t}^{j}+\left[f^{i j}\left(x_{t}\right) \eta_{t}^{j}+g^{i}(t)\right] d t, \quad t \in[0, T] \\
\eta_{0}^{i} & =0 .
\end{aligned}
$$

Then there exists a solution $x^{s}$ in $C_{o}(M)$ to the flow equation

$$
\begin{aligned}
\frac{d x^{s}}{d s} & =V\left(x^{s}\right), \quad s \in \mathbf{R} \\
x^{0} & =x
\end{aligned}
$$

where $x$ is the process in equation (1).

Furthermore, the paths $x^{s}$ are semimartingales of the form

$$
d x_{t}^{s}=X_{i}(s, t) \circ d w_{i}+X_{0}(s, t) d t
$$

where $X_{j}(s,),. 0 \leq j \leq n$ are adapted processes (with respect to $w$ ) in $T M$ such that, on a set of full Wiener measure, $s \mapsto X_{j}(s,$.$) is continuous into the space$ $L^{2}[0, T]$.

We prove Theorem 2 using a version of the Picard iteration scheme. The proof draws upon the closure of the class of Itô processes under composition with smooth maps, together with standard estimates on stochastic integrals. Each $m$-th stage of the iteration yields a family of Itô processes $d x_{t}^{s, m}=X_{i}^{m}(s, t) \circ d w_{i}+X_{0}^{m}(s, t) d t$. Expressions are obtained for the coefficients $X_{j}^{m}, 0 \leq j \leq n$, in terms of $\left\{X_{j}^{m-1}, 0 \leq\right.$ $j \leq n\}$. These expressions are used to prove that $X_{j}^{m}(s, t)$ converge to limits $X_{j}(s, t)$ that have the stability in $s$ described in the Theorem. The iterative procedure is then shown to imply that the processes $x^{s}$ defined by (3) satisfy the flow equation (2).

The following result is proved in [3]

Theorem 3 Suppose $N$ is a manifold equipped with a finite Borel measure $\gamma$. Let $Z$ be an admissible vector field on $N$ with flow $\left\{\sigma_{s}, s \in \mathbf{R}\right\}$ and let $\gamma_{s}$ denote the measure $\sigma_{s}(\gamma)$. Suppose there exists $B \subseteq N$ with $\gamma_{s}(B)=1$ for all $s$, such that Div $(Z)$ is defined and $Z$-differentiable on $B$, and that the function $s \mapsto \operatorname{Div}(Z)\left(\sigma_{s}(p)\right)$ is absolutely continuous for $p \in B$. Then $\gamma_{s}$ and $\gamma$ are equivalent measures and

$$
\frac{d \gamma_{s}}{d \gamma}(p)=\int_{0}^{s} \operatorname{Div}(Z)\left(\sigma_{-u}(p)\right) d u
$$


Combining Theorems 1,2 , and 3 , we obtain our main result

Theorem 4 Let $x^{s}$ denote the flow in Theorem 2 generated by the vector field $Z$ in Theorem 1. Then for each s, the law $\nu_{s}$ of $x^{s}$ is equivalent to $\nu$ and

$$
\frac{d \nu_{s}}{d \nu}(x)=\exp \int_{0}^{s} \operatorname{Div}(Z)\left(x^{-u}\right) d u
$$

Remark. Theorem 4 is similar to a result in Bruce Driver's paper [5]. However, the methods are different. Driver studied the class of vector fields on $C_{o}(M)$ obtained from Cameron-Martin paths in $T_{o} M$ by stochastic parallel translation along the paths of the diffusion process $x$. He constructed quasi-invariant flows for these

vector fields, then used the quasi-invariance property to deduce admissibility of the vector fields. In the work described here, this approach is reversed. We first construct a class of admissible vector fields, then show that they generate flows and quasi-invariant measures on $C_{o}(M)$.

In [7], Hu, Üstünel and Zakai studied rotation type measure-preserving transformations of abstract Wiener space and constructed flows associated to these transformations. In contrast to the flows studied here and in Driver's work, the transformations considered in [7] are non-adapted (the notion of adaptedness having no obvious meaning in the context of an abstract Wiener space).

\section{References}

[1] D. Bell, Divergence theorems in path space. J. Funct. Anal. 218 (2005), no. 1, $130-14$.

[2] D. Bell, Divergence theorems in path space II: degenerate diffusions, C. R. Acad. Sci. Paris Sr. I Math., to appear.

[3] D. Bell, Admissible vector fields and quasi-invariant measures. Appendix to The Malliavin Calculus, 2nd edition. Dover Publications, Mineola, NY, 2006.

[4] A. B. Cruzeiro, Équations différentielles sur l'espace de Wiener et formules de Cameron-Martin non-linéaires. J. Funct. Anal. 54 (1983) 206-227.

[5] B. Driver, A Cameron-Martin type quasi-invariance theorem for Brownian motion on a compact manifold. J. Funct. Anal. 109 (1992) 272-376.

[6] E. P. Hsu, Quasi-invariance of the Wiener measure on the path space over a compact Riemannian manifold. J. Funct. Anal. 134 (1995) 417-450.

[7] Y. Hu, A. S. Üstünel and M. Zakai, Tangent processes on Wiener space. J. Funct. Anal. 192 (2002), no. 1, 234-270. 